\title{
Compound-Transporter Interaction Studies using Canonical Correlation Analysis
}

\author{
Masato Kitajima ${ }^{1,2}$, Yohsuke Minowa ${ }^{3}$, Hideo Matsuda ${ }^{2}$, Yasushi Okuno ${ }^{4 *}$ \\ ${ }^{1}$ Current Address:Life Science Systems Dept. ,PLM Solutions Div. \\ ,Fujitsu Kyushu System Engineering Limited, \\ 2-2-1,Momochihama,Sawara-ku,Fukuoka,814-8589,Japan \\ ${ }^{2}$ Department of Bioinformatic Engineering, Graduate School of Information Science and Technology, \\ Osaka University, 1-3 Machikaneyama, Toyonaka, Osaka, 560-8531, Japan, \\ ${ }^{3}$ National Institute of Biomedical Innovation \\ Toxicogenomics Informatics Project \\ 7-6-8 Asagi Saito Ibaraki-City Osaka, 567-0085,Japan \\ ${ }^{4}$ Department of PharmacoInformatics, Center for Integrative Education of \\ Pharmacy Frontier, Graduate School of Pharmaceutical Sciences, Kyoto University \\ 46-29 Yoshida-Shimo-Adachi-cho, Sakyo-ku, Kyoto 606-8501, Japan \\ *E-mail: okuno@pharm.kyoto-u.ac.jp
}

(Received November 3, 2007; accepted November 15, 2007; published online December 5, 2007)

\begin{abstract}
The efficient screening of lead compounds or drug candidates for efficacy and safety is critically important during the early stage of drug development. Compounds are usually screened from a diverse 'chemical space' based only on its pharmacological effects, but this screening is not enough to guarantee drug safety. To solve this problem, we devised a chemical space that takes into account interaction information with proteins such as drug transporters. We also created and evaluated a mathematical model for predicting compound-transporter interactions. This was achieved by first generating an interaction correlation matrix based on drug transporters and their corresponding inhibitor compounds. To implement a screening scheme that takes into account interaction with drug transporters, we created a model using Canonical Correlation Analysis (CCA) that makes use of the known information on interaction between drug transporters and their corresponding inhibitors. Cross-validation of the model gave satisfactory test results and a physiologically relevant chemical space was constructed based on the model.
\end{abstract}

Key Words: Pharmacokinetics, Transporter, chemoinformatics, bioinformatics

Area of Interest: Molecular Recognition 


\section{Introduction}

During the drug development process it is important to screen compounds for efficacy and safety at an early stage in order to prevent unnecessary and costly analysis later on. It is thought that the diverse chemical space may contain as much as $10^{60}$ chemical structures or more. Searching for a drug candidate with a good balance of efficacy and safety from this huge chemical space is obviously very difficult, as evidenced from the fall in the number of new drug applications in recent years [1][2].

The previously used screening approach involved sampling a diverse chemical library for those leads that display only promising pharmacological effects i.e. drug efficacy. It is important to select a compound with excellent pharmacokinetic properties (drug safety), not just pharmacological effects, when screening at the early stage of drug development. When analyzing the pharmacokinetic properties of drug candidates, ligand interactions with Phase I enzymes such as cytochromes P450, Phase II conjugation enzymes (e.g. GST and sulfotransferases), as well as transporter proteins that play a crucial role in Phase III, must be considered [3]. Of these, transporter proteins, which are important in facilitating absorption of compounds in the intestines as well as the degree of penetration across the blood-brain barrier, play a central role in determining the bioavailability of drugs.

This paper focuses on transporter proteins that play an important role in drug pharmacokinetics. The interaction studies were carried out based on information on the interactions of the compounds and the transporter proteins. To implement a screening scheme that takes into account interaction with drug transporters, we created a model using CCA that exploits the characterized interactions between drug transporters and their corresponding inhibitors. The model is evaluated and then used to create a physiologically relevant chemical space.

\section{Method}

\subsection{Gathering of compound-transporter interaction data}

The compound-transporter interaction data used in this study was extracted from the ADME Database (developed by Fujitsu Kyushu Systems Engineering Ltd., Fukuoka, Japan) [4][5][6]. The database is a collection of information on drug transporters as well as drug metabolizing enzymes found in the literature. Two kinds of transporter proteins were selected for this study; the ABC transporter family and the SLC transporter family. Compounds that interact with these transporter families were also extracted from the database. The compound-transporter interaction type available in the database includes substrates, inhibitors, inducers and activators. However, for the purpose of this study only the inhibitors were selected.

A total of $17 \mathrm{ABC}$ transporter families and 110 different SLC transporter proteins were selected for this study. Data concerning the interaction of these transporter proteins with known compounds was extracted from the ADME Database. The database contains 5,860 compound-transporter interactions between the selected 117 transporter proteins and their interacting 3,275 compounds.

The selected transporter proteins are as follows. 
Table 1. List of transporter proteins used in the study

\begin{tabular}{llllll}
\hline ABCA1 & SLC1A1 & SLC5A6 & SLC7A7 & SLC19A1 & SLC23A1 \\
ABCA2 & SLC1A2 & SLC5A7 & SLC7A8 & SLC19A2 & SLC23A2 \\
ABCA9 & SLC1A3 & SLC5A8 & SLC10A1 & SLC21A11 & SLC26A2 \\
ABCA10 & SLC1A4 & SLC5A9 & SLC10A2 & SLC21A12 & SLC26A3 \\
ABCB1 & SLC1A5 & SLC6A1 & SLC10A4 & SLC21A14 & SLC26A4 \\
ABCB4 & SLC1A6 & SLC6A11 & SLC13A1 & SLC21A2 & SLC26A6 \\
ABCB5 & SLC1A7 & SLC6A12 & SLC13A2 & SLC21A20 & SLC26A7 \\
ABCB11 & SLC2A1 & SLC6A13 & SLC13A3 & SLC21A3 & SLC26A8 \\
ABCC1 & SLC2A10 & SLC6A14 & SLC13A4 & SLC21A6 & SLC26A9 \\
ABCC2 & SLC2A11 & SLC6A2 & SLC13A5 & SLC21A8 & SLC27A4 \\
ABCC3 & SLC2A12 & SLC6A3 & SLC15A1 & SLC21A9 & SLC28A1 \\
ABCC4 & SLC2A13 & SLC6A4 & SLC15A2 & SLC22A1 & SLC28A2 \\
ABCC5 & SLC2A2 & SLC6A5 & SLC15A4 & SLC22A11 & SLC28A3 \\
ABCC10 & SLC2A3 & SLC6A6 & SLC16A1 & SLC22A12 & SLC29A1 \\
ABCC11 & SLC2A4 & SLC6A9 & SLC16A10 & SLC22A16 & SLC29A2 \\
ABCG1 & SLC2A6 & SLC7A1 & SLC16A3 & SLC22A2 & SLC29A4 \\
ABCG2 & SLC2A7 & SLC7A10 & SLC16A5 & SLC22A3 & SLC32A1 \\
& SLC2A8 & SLC7A11 & SLC16A7 & SLC22A4 & SLC36A1 \\
& SLC4A4 & SLC7A2 & SLC17A1 & SLC22A5 & SLC38A1 \\
& SLC5A1 & SLC7A3 & SLC18A1 & SLC22A6 & SLC38A4 \\
& SLC5A2 & SLC7A5 & SLC18A2 & SLC22A7 & SLC38A5 \\
& SLC5A4 & SLC7A6 & SLC18A3 & SLC22A8 & SLC43A2 \\
\hline
\end{tabular}

\subsection{Organizing the collected data}

A correlation matrix of compound-transporter interactions was constructed based on the collected compound-transporter interaction information. Here compounds that interact with a transporter protein are flagged ' 1 ', and those that do not interact are flagged ' 0 ' as shown in Table2.

The similarity between transporter proteins was calculated based on this interaction correlation matrix. The Tanimoto coefficient found below was used to evaluate similarity [7] .

$$
\begin{aligned}
& \text { Tanimoto }(X, Y)=\frac{C}{A+B-C} \\
& \text { A: No. of bits in } X \text { that were flagged ' } 1 \text { ' } \\
& \text { B: No. of bits in } Y \text { that were flagged ' } 1 \text { ' } \\
& \text { C: No. of flagged bits common to both } X \text { and } Y
\end{aligned}
$$

The interaction similarity of the compounds was also defined as Tanimoto coefficient based on the correlation matrix. We used the distance measure transformed from the Tanimoto coefficient as shown below:

$$
\operatorname{Distance}(X, Y)=1-\operatorname{Tanimoto}(X, Y)
$$


Table 2. Excerpt from the correlation matrix of compound-transporter interactions.

\begin{tabular}{|c|c|c|c|c|c|c|c|c|c|c|c|c|c|c|c|c|c|}
\hline Transporter Name & 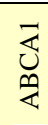 & 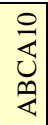 & 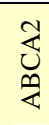 & 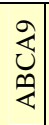 & $\begin{array}{l}\overline{0} \\
0 \\
\bar{q}\end{array}$ & 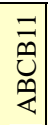 & 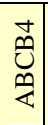 & $\begin{array}{l}n \\
0 \\
0 \\
0\end{array}$ & $\begin{array}{l}\bar{U} \\
\bar{\otimes} \\
z\end{array}$ & \begin{tabular}{l|}
0 \\
$ن$ \\
$u$ \\
0 \\
$<$
\end{tabular} & 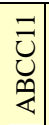 & 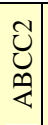 & 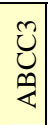 & 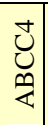 & $\begin{array}{l}\tilde{U} \\
己 \\
0 \\
z\end{array}$ & $\begin{array}{l}\bar{J} \\
\bar{x} \\
\bar{\psi}\end{array}$ & 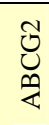 \\
\hline Verapamil & 0 & 0 & 0 & 0 & 0 & 1 & 1 & 0 & 1 & 1 & 0 & 1 & 1 & 1 & 0 & 0 & 0 \\
\hline Cholyltaurine, Taurocholic acid, Taurocholate & 0 & 0 & 0 & 0 & 0 & 1 & 0 & 0 & 1 & 1 & 0 & 0 & 1 & 1 & 0 & 0 & 1 \\
\hline 4,4'-Diisothiocyanostilbene-2,2'-disulfonic acid, DIDS & 1 & 0 & 0 & 0 & 0 & 0 & 0 & 0 & 1 & 0 & 1 & 0 & 0 & 0 & 1 & 0 & 0 \\
\hline Phloretin & 0 & 0 & 0 & 0 & 1 & 0 & 0 & 0 & 1 & 0 & 0 & 0 & 0 & 0 & 0 & 0 & 1 \\
\hline Bromosulfophthalein, Sulfobromophthalein, BSP & 1 & 0 & 0 & 0 & 0 & 0 & 0 & 0 & 0 & 0 & 0 & 1 & 0 & 1 & 1 & 0 & 0 \\
\hline Cyclosporin A, Cyclosporine, Cyclosporin, Ciclosporin & 0 & 0 & 0 & 0 & 1 & 1 & 1 & 0 & 1 & 1 & 0 & 1 & 1 & 1 & 0 & 1 & 1 \\
\hline Probenecid & 0 & 0 & 0 & 0 & 0 & 0 & 0 & 0 & 1 & 1 & 0 & 1 & 1 & 1 & 1 & 0 & 0 \\
\hline Indomethacin & 0 & 0 & 0 & 0 & 1 & 0 & 0 & 0 & 1 & 0 & 0 & 1 & 1 & 1 & 0 & 0 & 0 \\
\hline Progesterone & 0 & 0 & 0 & 0 & 1 & 1 & 0 & 0 & 1 & 0 & 0 & 1 & 0 & 1 & 1 & 0 & 1 \\
\hline Quinidine & 0 & 0 & 0 & 0 & 1 & 0 & 0 & 0 & 1 & 0 & 0 & 0 & 0 & 1 & 0 & 0 & 0 \\
\hline Cimetidine & 0 & 0 & 0 & 0 & 1 & 0 & 0 & 0 & 0 & 0 & 0 & 0 & 0 & 0 & 0 & 0 & 0 \\
\hline Phloridzin, Phlorizin & 0 & 0 & 0 & 0 & 1 & 0 & 0 & 0 & 0 & 0 & 0 & 0 & 0 & 0 & 0 & 0 & 0 \\
\hline Pravastatin, Pravastatin acid & 0 & 0 & 0 & 0 & 1 & 1 & 0 & 0 & 0 & 0 & 0 & 1 & 0 & 0 & 0 & 0 & 1 \\
\hline Rifampicin, Rifampin & 1 & 0 & 0 & 0 & 1 & 1 & 0 & 0 & 1 & 0 & 0 & 1 & 1 & 0 & 0 & 0 & 1 \\
\hline 1-Methyl-4-phenylpyridinium, MPP(+) & 0 & 0 & 0 & 0 & 1 & 0 & 0 & 0 & 0 & 0 & 0 & 0 & 0 & 0 & 0 & 0 & 0 \\
\hline Estradiol 17beta-D-glucuronide, E(2)17betaG & 0 & 0 & 0 & 0 & 0 & 0 & 0 & 0 & 1 & 0 & 0 & 1 & 1 & 1 & 1 & 0 & 0 \\
\hline L-glutamine, Gln & 0 & 0 & 0 & 0 & 0 & 0 & 0 & 0 & 0 & 0 & 0 & 0 & 0 & 0 & 0 & 0 & 0 \\
\hline L-leucine, L-Leu & 0 & 0 & 0 & 0 & 0 & 0 & 0 & 0 & 0 & 0 & 0 & 0 & 0 & 0 & 0 & 0 & 0 \\
\hline Methotrexate & 0 & 0 & 0 & 0 & 1 & 0 & 0 & 0 & 0 & 1 & 0 & 1 & 1 & 1 & 1 & 0 & 1 \\
\hline MK571, MK-571 & 0 & 0 & 0 & 0 & 1 & 0 & 0 & 0 & 1 & 1 & 0 & 1 & 1 & 1 & 1 & 0 & 1 \\
\hline Chlorpromazine & 0 & 0 & 0 & 0 & 1 & 1 & 0 & 0 & 0 & 0 & 0 & 0 & 0 & 0 & 0 & 0 & 0 \\
\hline Desimipramine, Desipramine & 0 & 0 & 0 & 0 & 1 & 0 & 0 & 0 & 0 & 0 & 0 & 0 & 0 & 0 & 0 & 0 & 0 \\
\hline Diclofenac & 0 & 0 & 0 & 0 & 0 & 0 & 0 & 0 & 1 & 0 & 0 & 0 & 0 & 1 & 0 & 0 & 0 \\
\hline Ketoprofen & 0 & 0 & 0 & 0 & 0 & 0 & 0 & 0 & 1 & 0 & 0 & 0 & 0 & 1 & 0 & 0 & 0 \\
\hline
\end{tabular}

\subsection{Canonical Correlation Analysis (CCA)}

Next, CCA was performed using the collected compound-transporter interaction data. Dragon X was used to calculate the compound descriptors [8]. A total of 929 descriptors were calculated. Highly correlated descriptors were grouped together and then filtered to give a final total of 324 compound descriptors.

The transporter proteins were calculated as bigram (two amino acids) frequency in protein sequences, and were used to generate a total of 400 protein descriptors. CCA was then performed, which generated 324 components. CCA is a technique to extract common features from a pair of multivariate data (chemical and protein descriptors). CCA finds a linear transformation of the chemical and protein spaces such that the correlation coefficient is maximized. Therefore we can construct the chemical space with the higher correlation to the protein space by extraction of the some components with the higher correlation coefficients.

\section{4 Cross Validation}

A 5-fold cross-validation test was performed using only the 44 CCA components with P value < 0.01 of correlation coefficient test. The whole training compound set was divided into five sets. The first set was left out for testing and the remaining four sets were used to train a model. The compounds from the first set were then used to evaluate the trained model. The model was evaluated by setting a Euclid distance threshold from a test compound and using the closest neighboring compounds within this threshold for prediction. The procedure was repeated for all five sets, each time leaving out one set for testing, until all compounds from all five sets had been evaluated. 


\section{Result}

\subsection{Analysis of compound-transporter interactions}

A significant number of compounds were found to inhibit more than one transporter using the collected compound-transporter interaction data. Indeed, out of these compounds, 183 were identified as inhibiting five or more transporters. The list below shows the frequency for each "interaction count" of a compound.

Table 3. Frequency of Interaction count for a single compound

\begin{tabular}{cc}
\hline Interaction Count & Frequency \\
\hline 18 & 1 \\
17 & 1 \\
16 & 2 \\
15 & 3 \\
14 & 1 \\
13 & 2 \\
12 & 4 \\
11 & 6 \\
10 & 8 \\
9 & 15 \\
8 & 19 \\
7 & 18 \\
6 & 37 \\
5 & 66 \\
4 & 118 \\
3 & 355 \\
2 & 393 \\
1 & 2226 \\
\hline
\end{tabular}

Moreover, the similarity of each transporter protein was calculated from the interaction matrix profile by using the Tanimoto coefficient. The result of clustering based on this similarity measure is shown in Figure 1. As shown in the figure, ABCG1 and ABCB4 are similar by interaction pattern even though the sequence similarity between both proteins is very low. Analogous results were found for ABCG2, which was shown to be similar to ABCC1 and ABCC2.

Moreover, Cyclosporin A that interacts with 15 kinds of transporter proteins, and MK571 that interacts with 11 kinds of transporter proteins were examined and compared as shown in Figure 2 [9][10]. Even though the two compounds share a low degree of structural similarity, they were found to interact with the same 10 transporter proteins. Our results demonstrate that it is not possible to explain all the similarities in interaction by simply comparing the structure of the relevant compounds or by protein sequence alignments alone. We then performed CCA on the collected interaction data to construct a correlation model for building a chemical space that reflected the classification of the transporters. 
Chem-Bio Informatics Journal, Vol. 7, No. 2, pp.24-34(2007)

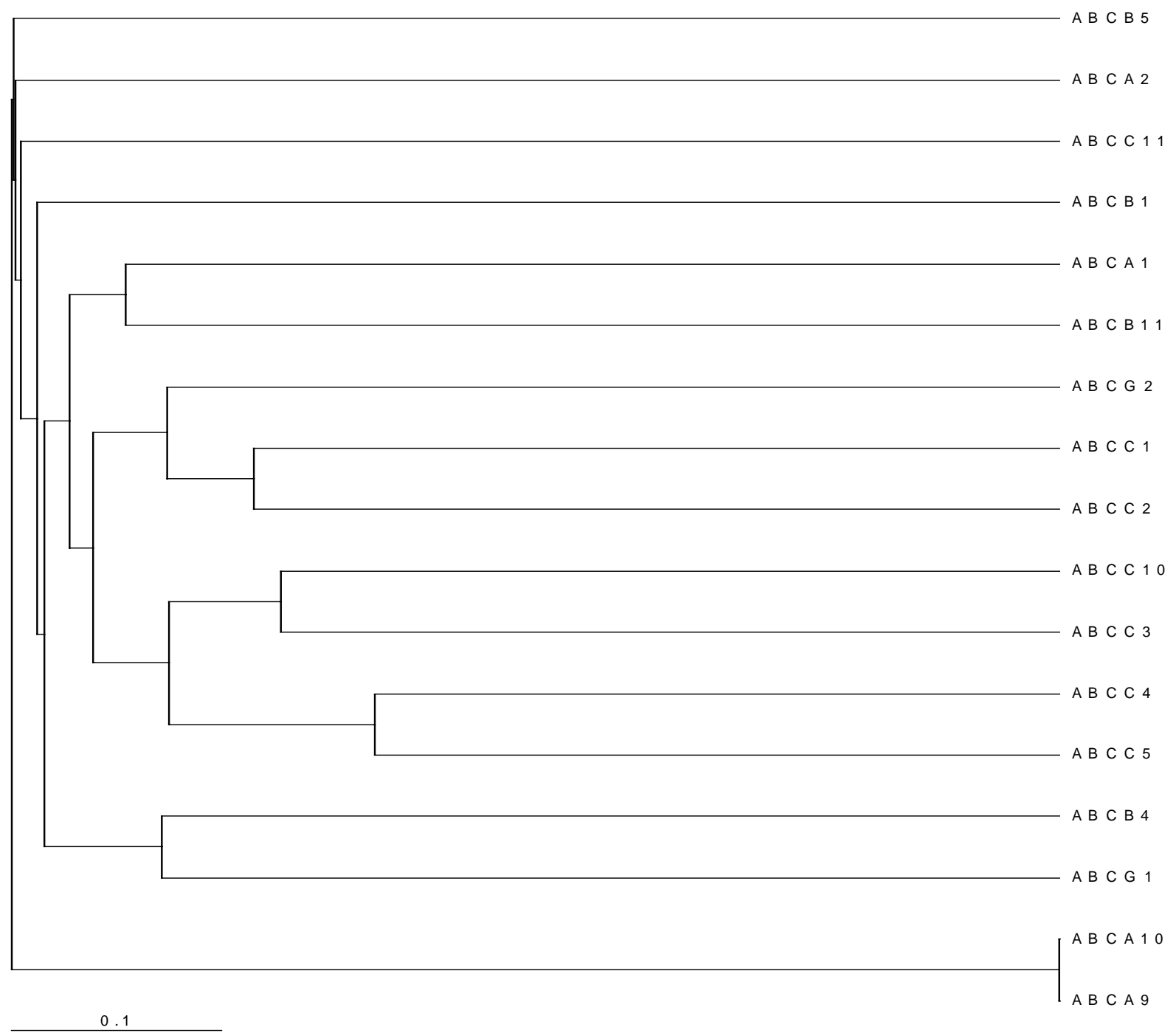

Figure 1. ABC transporters' cluster analysis based on similarity of interaction 
<smiles>CCNC(=O)CCSC(SCCC(=O)O)c1cccc(/C=C/c2ccc3ccc(Cl)cc3n2)c1</smiles>

MK571<smiles></smiles>

Cyclosporin A

Figure2. Chemical structures of MK571 and CyclosporinA

\subsection{CCA Result}

The correlation model was constructed by using canonical correlation analysis (CCA). Performance of the model was evaluated using 5-fold cross-validation. CCA analysis and 5-fold cross-validation were performed using the collected compound-transporter interaction data. Below is the definition of the terms used in the evaluation of results.

Table 4. Definition of terms used in validation results

List of the total number of compounds (frequency) with corresponding numbers of transporter interactions (interaction count)

\begin{tabular}{|c|l|}
\hline TruePositive & $\begin{array}{l}\text { Definition } \\
\text { The predicted transporter-interaction matches an observed } \\
\text { transporter-interaction of the test compound }\end{array}$ \\
\hline TrueNegative & $\begin{array}{l}\text { The predicted NON- interaction matches an observed } \\
\text { NON-interaction of the test compound }\end{array}$ \\
\hline FalsePositive & $\begin{array}{l}\text { The predicted transporter-interaction do not match any of the } \\
\text { observed transporter-interaction of the test compound }\end{array}$ \\
\hline FalseNegative & $\begin{array}{l}\text { The predicted NON- interaction matches an observed } \\
\text { transporter-interaction of the test compound }\end{array}$ \\
\hline
\end{tabular}


Chem-Bio Informatics Journal, Vol. 7, No. 2, pp.24-34(2007)

Sensitivity and Specificity are calculated as follows:

Sensitivity $=\frac{\text { TruePositive }}{\text { TruePositive }+ \text { FalseNegative }}$

Specificity $=\frac{\text { TrueNegative }}{\text { TrueNegative }+ \text { FalsePositive }}$

FalsePositiveRate=1-Specificity

To evaluate the performance of the model, the ROC plot ( $x$-axis=FalsePositiveRate, $\mathrm{y}$-axis=Sensitivity) is shown in Figure 3 below.

Transporter inhibitor ROC

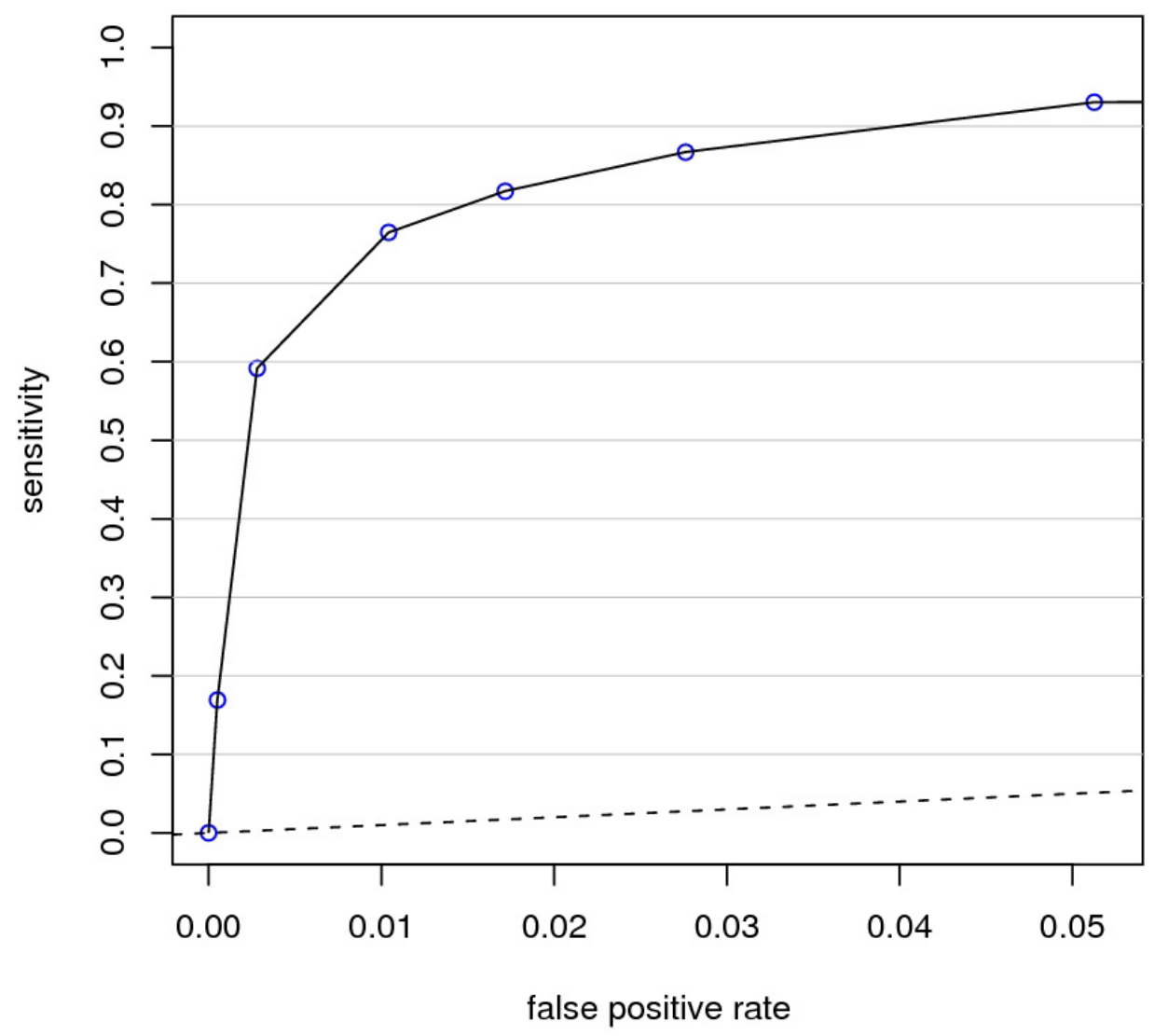

Figure 3. ROC of transporter inhibitor

ROC was plotted the average point of the sensitivity and false positive rate calculated under each condition of the maximum number of neighboring compounds $(1,5,10,20)$ and the Euclid distance thresholds $(1,10,20,40,80,160$ and 200) 
This graph shows that the closer the curve inclines to the upper left corner the better is the model performance. The closer the curve declines to the dotted line the poorer is the performance, since the dotted line shows the performance curve of random models. The results show that our model has a very high performance, as evidenced by the curve's inclination to the upper left corner.

Then we identified 183 compounds that interact with more than 5 transporter proteins. A similarity map of the compounds was constructed as shown in Figure 4; where x-axis represents the similarity based on structural descriptors and y-axis represents similarity based on the interaction correlation matrix (Table 1). Our similarity map shows two distinct groups of compounds. Group B represents compounds with extremely low structural descriptor similarity, as exemplified by Cyclosporin A and Vinblastine. In this group, Cyclosporin A and MK571 show relatively high interaction similarity even though they have low structural descriptor similarity. For clarity, the similarity map of group A is enlarged by excluding group B as shown in Figure 5. The figure also shows that even though the compounds which interact with the subfamily of SLC22 and SLC28 have low structural similarity, they show high similarity with regards to interaction with SLC22 or SLC28.

Furthermore, the same similarity map is reconstructed by plotting in the $\mathrm{x}$-axis the similarity of the compounds measured by CCA (instead of using the structural descriptors) as shown in Figure 6. The figure shows that compounds with low structural similarity may have high similarity by CCA, as shown by the Cyclosporin A and MK571 pair, as well as the SLC22 and the SLC28 interacting compounds. Thus, it was shown that constructing a chemical space using information on compound-transporter interaction is much better than simply using structural descriptors alone.

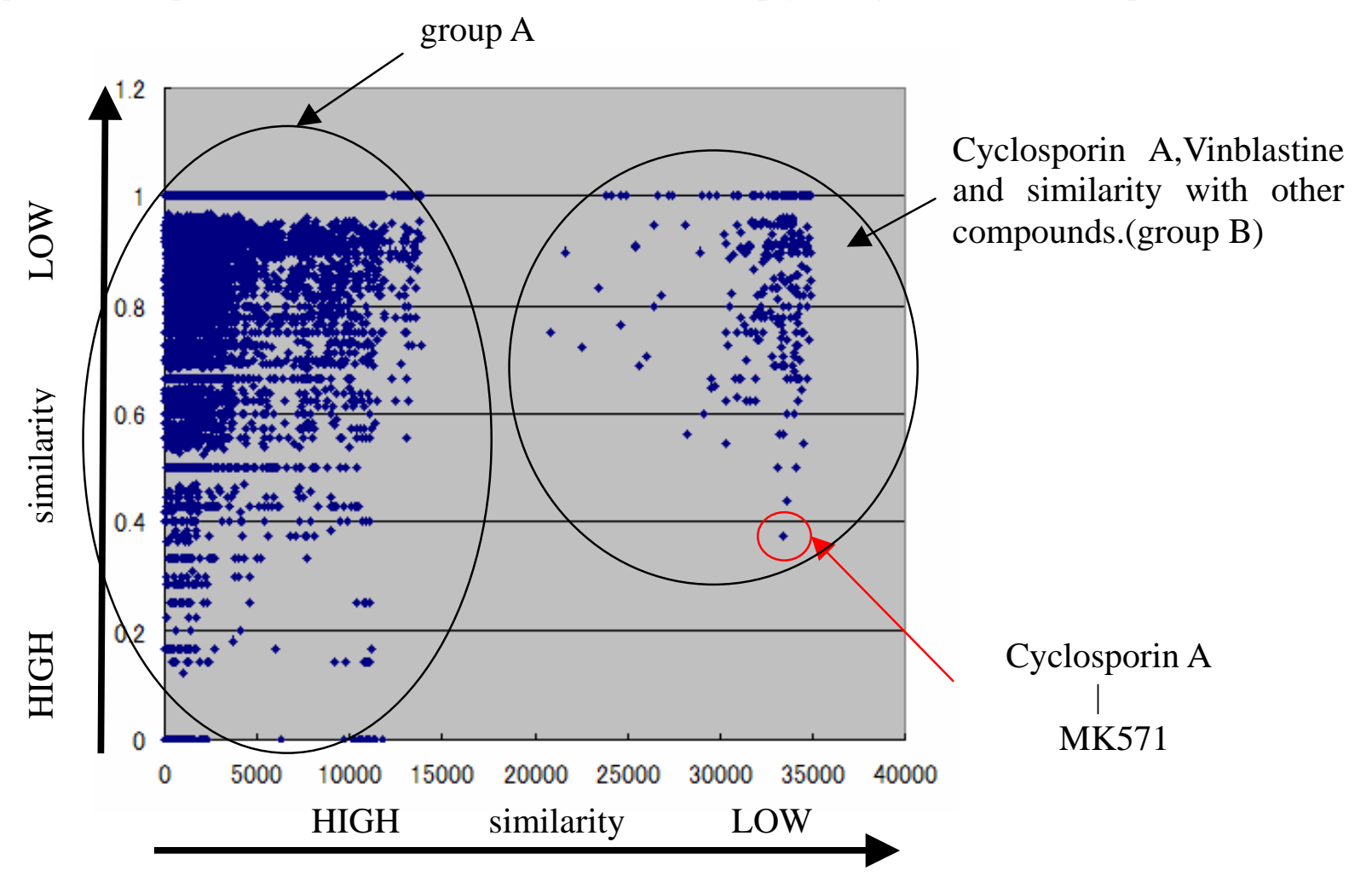

Figure 4. Two-dimensional map of structural similarity vs. interaction similarity

(Cyclosporin A and Vinblastine included in the map) $\mathrm{y}$-axis : Similarity in interaction pattern with a transporter protein $\mathrm{x}$-axis : Similarity in chemical structure 


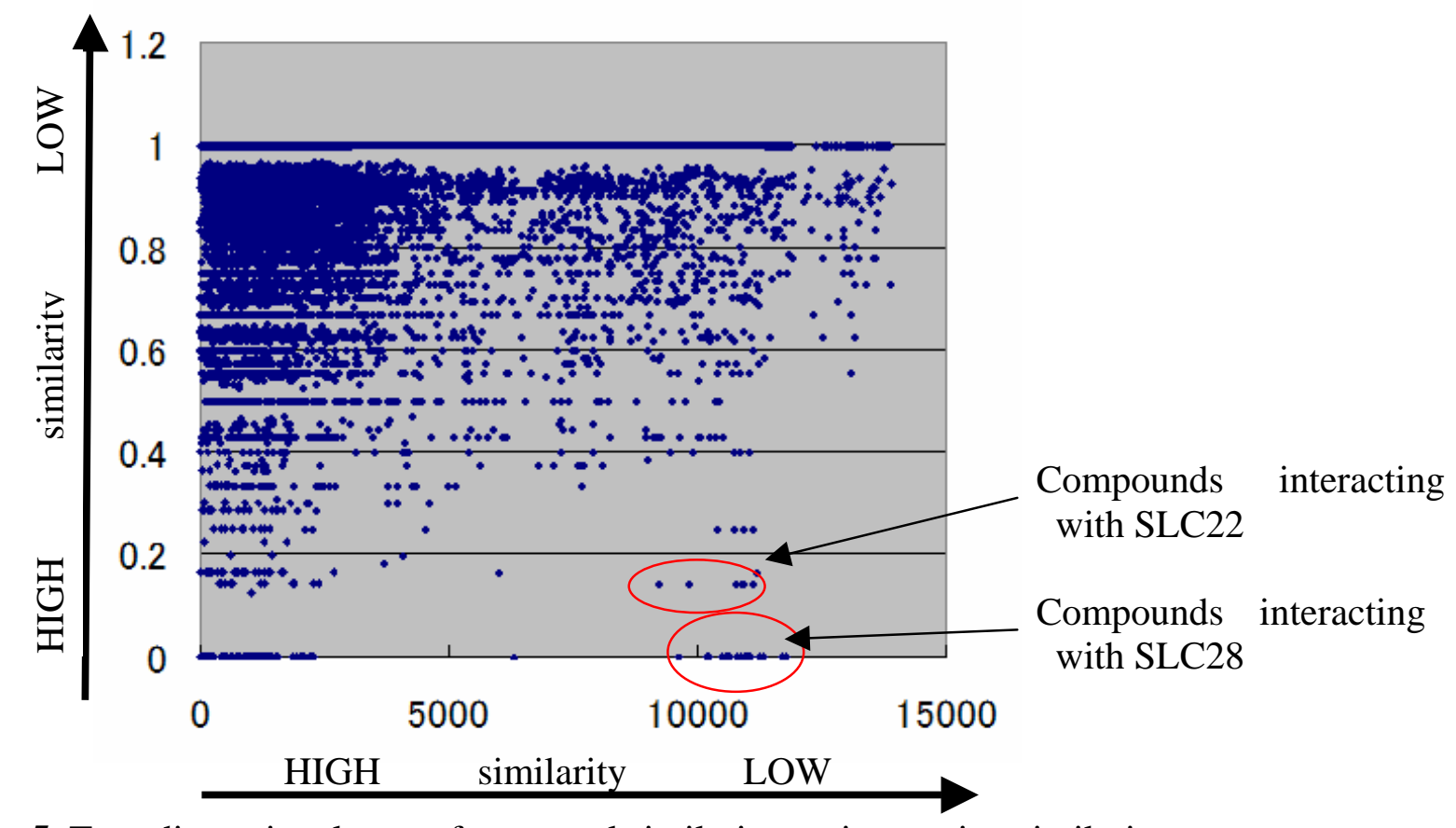

Figure 5. Two-dimensional map of structural similarity vs. interaction similarity

(Cyclosporin A and Vinblastine excluded from the map) $y$-axis : Similarity in interaction pattern with a transporter protein $\mathrm{X}$-axis : Similarity in chemical structure

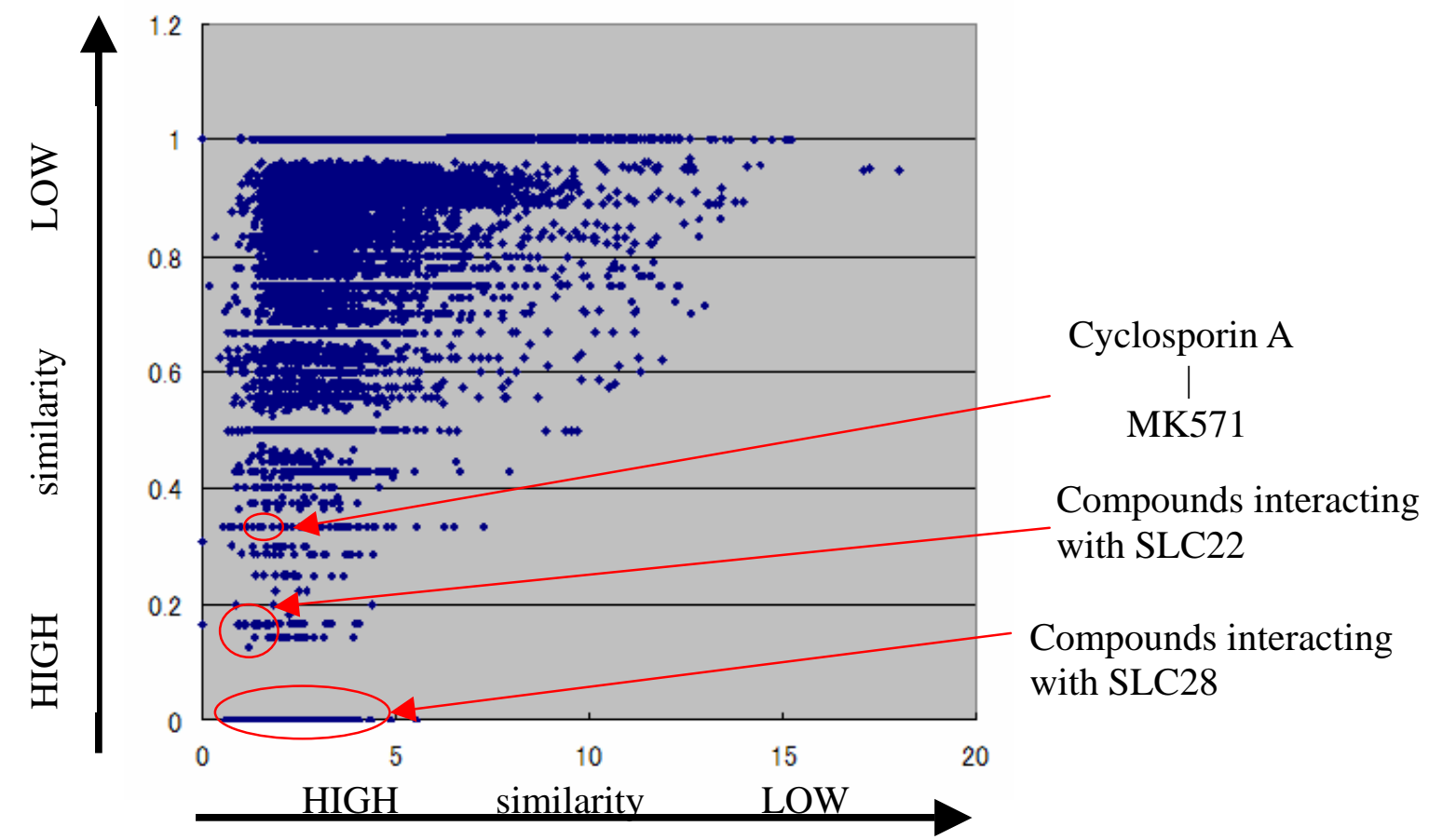

Figure 6. Two-dimensional map of similarity by CCA vs. interaction similarity

(Cyclosporin A and Vinblastine included in the training) $y$-axis : Similarity in interaction with a transporter protein $\mathrm{x}$-axis : Similarity by CCA 


\section{Discussion}

It was found that the results of classifying the transporter proteins by similarity of interaction pattern are different from the results obtained when classifying them by sequence similarity. Moreover, it was found that compounds showing similarity in interactions with more than one transporter protein may not necessarily have structural similarity at all.

These results show that it is difficult to predict the interaction between a compound and protein (i.e. related to pharmacological and pharmacokinetic effects) based on chemical structural similarity or protein sequence similarity alone. To create a physiologically relevant chemical space, information on compound-protein interactions is required. By utilizing CCA, we built an interaction model that was used to create a chemical space. Compounds that have high similarity in terms of interaction with proteins, but that are not necessarily similar in terms of structure, were clustered together. Thus a chemical space of transporter inhibitors was created, although this technique can also be applied to construct a chemical space (or a focused library) of compounds that interact with any specific target protein.

Moreover, a compound-transporter interaction model was constructed using CCA, which gave good evaluation results. This technique can be extended to develop chemical spaces of not only the inhibitors but also the substrates of transporter proteins. The resulting chemical spaces may be used for in silico screening of compounds with good pharmacological characteristics as well as good absorption, distribution and excretion properties.

The method described in this paper can also be extended to study toxicity related proteins. By building chemical spaces for such proteins, drug candidates with a good balance of efficacy and safety can be developed.

\section{References}

[1] Peter Kirkpatrick and Clare Ellis, Chemical space, Nature, 432, 823 (2004).

[2] Christopher M. Dobson, Chemical space and biology, Nature, 432, 824-828 (2004).

[3] Ishikawa T. The ATP-dependent glutathione S-conjugate export pump, Trends Biochem. Sci., 17, 463-468 (1992).

[4] http://jp.fujitsu.com/group/fqs/services/lifescience/asp/adme-database/index.html

[5] Rendic S., Summary of information on human CYP enzymes: human P450 metabolism data. Drug Metab. Rev., 34, 83-448 (2002).

[6] Rendic S, Di Carlo F.J., Human cytochrome P450 enzymes: a status report summarizing their reactions, substrates, inducers, and inhibitors. Drug Metab. Rev., 29, 413-580 (1997).

[7] Godden J.W., Xue L., Bajorath J., Combinatorial Preferences Affect Molecular Similarity/Diversity Calculations Using Binary Fingerprints and Tanimoto Coefficients, Journal of Chemical Information and Computer Sciences, 40, 163-166 (2000).

[8] http://www.talete.mi.it/products/dragon_description.htm

[9] Byrne JA, Strautnieks SS, Mieli-Vergani G, Higgins CF, Linton KJ, Thompson RJ. The human bile salt export pump: characterization of substrate specificity and identification of inhibitors, Gastroenterology, 123, 1649-1658 (2002).

[10] Leier I, Jedlitschky G, Buchholz U, Center M, Cole SP, Deeley RG, Keppler D. ATP-dependent glutathione disulphide transport mediated by the MRP gene-encoded conjugate export pump, Biochem. J., 314, 433-437 (1996). 